\title{
Efecto de amortiguamiento térmico de una barrera verde de Arundo donax como elemento de bioclimatización en edificios
}

\section{Thermal damping effect due to a green barrier which includes Arundo donax as bioclimatic element in buildings}

\author{
$\underline{\text { P. Rodríguez-Salinas }}^{(*)}$, M. Ruiz Morales ${ }^{(* *)}$, A. Franco ${ }^{(*)}$, A. R. Pérez-Fernández ${ }^{(*)}$, O. Lobato-Calleros ${ }^{(* *)}$
}

RESUMEN

Entre los principales impactos ambientales derivados de la operación de edificios habitacionales está la generación de gases de efecto invernadero resultante del consumo eléctrico de sistemas de climatización. El uso de sistemas de vegetación representa una alternativa para disminuir dicho consumo energético. Las barreras verdes son sistemas de vegetación empleados como protección contra el viento, aunque recientemente se ha explorado su uso como sistemas de amortiguación acústica. En este trabajo se analiza su posible aplicación como sistemas de aislamiento térmico para edificios. En específico, se reporta el comportamiento de una barrera verde de Arundo donax como elemento de bioclimatización. Los resultados se analizan a partir de las temperaturas medidas en el interior y exterior de edificios prototipo, en función de la presencia de una barrera verde. Adicionalmente se determinó la traspiración del Arundo donax en condiciones ambientales extremas.

Palabras clave: arquitectura bioclimática; Arundo donax; barrera verde; amortiguamiento térmico.

\section{ABSTRACT}

Among the main environmental impacts of the operation of residential buildings are those due to greenhouse gases generation as a result of electric consumption of air conditioning systems. The use of vegetation systems in residential buildings represents an alternative to reduce this energy consumption. Green vegetation systems barriers are often used as protection against winds, but recently they are also being used as acoustic dampers. This work explores their use as thermal insulation systems for buildings. Specifically, we report the behavior of an Arundo donax green barrier as a bioclimatic element. The results are analyzed based on indoor and outdoor temperature measurement in prototype buildings, in function of the green barrier presence. Additionally Arundo donax transpiration under extreme environmental conditions was determined.

Keywords: bioclimatic architecture; Arundo donax; green barrier; thermal insulation.

(*) División Académica de Tecnología Ambiental y Nanotecnología, Universidad Tecnológica Fidel Velázquez. Ciudad Nicolás Romero (México).

${ }^{(* *)}$ Departamento de Ingenierías, Universidad Iberoamericana. Ciudad de México (México).

Persona de contacto/Corresponding author: perla.rodriguez@utfv.edu.mx (P. Rodríguez-Salinas)

ORCID: http://orcid.org/oooo-0002-8440-8024 (P. Rodríguez-Salinas); http://orcid.org/oooo-0002-0456-1038

(M. Ruiz Morales); http://orcid.org/oooo-0oo3-1803-6258 (A. Franco); http://orcid.org/oooo-0002-3085-7174

(A.R. Pérez-Fernández); http://orcid.org/oooo-0oo1-8756-7840 (O. Lobato-Calleros).

Cómo citar este artículo/Citation: Rodríguez-Salinas, P., Ruiz Morales, M., Franco, A., Pérez-Fernández, A. R., Lobato-Calleros, O. (2017). Efecto de amortiguamiento térmico de una barrera verde de Arundo donax como elemento de bioclimatización en edificios. Informes de la Construcción, 69(547): e216, doi: http://dx.doi.org/10.3989/ic.16.127.

Copyright: (C) 2017 CSIC. Licencia / License: Salvo indicación contraria, todos los contenidos de la edición electrónica de Informes de la Construcción se distribuyen bajo una licencia de uso y distribución Creative Commons Attribution License (CC BY) Spain 3.o. 


\section{INTRODUCCIÓN}

La excesiva dependencia de los combustibles fósiles como energéticos es actualmente, desde el punto de vista de protección ambiental, uno de los aspectos más importantes por resolver. El consumo energético dedicado a controlar el confort ambiental en espacios cerrados a través de sistemas de aire acondicionado constituye una fuerte preocupación ambiental, debido principalmente a los efectos dañinos que ocasionan los gases de efecto invernadero generados, como consecuencia del consumo eléctrico de su operación.

Siendo un país con una variedad climática muy contrastante, con ecosistemas que abarcan desde el bosque y selva tropical hasta el desierto, México enfrenta retos importantes en materia de racionalización del consumo energético destinado a la climatización artificial. Durante primavera y verano, en el noreste, noroeste, sur y sureste de México son frecuentes los días con temperaturas máximas iguales o superiores a $40^{\circ} \mathrm{C}$ (Figura 1) (1).

La cantidad de energía utilizada en sistemas de climatización en un hogar ubicado en una región con clima extremoso es prácticamente el doble de la consumida en clima templado o cálido (Figura 2). Este gasto representa no sólo una importante erogación para las familias, sino un fuerte impacto ambiental.

Con la finalidad de contrarrestar estos impactos negativos en los últimos años se han desarrollado diversas «estrategias arquitectónicas bioclimáticas», cuyo objetivo básico es generar ambientes con temperaturas que resulten más agradables, empleando elementos que generen, acumulen y transmitan calor (2).

Dentro de estas estrategias el uso de infraestructura verde es cada vez más común, y aunque no existe consenso sobre una clasificación general para ésta, se pueden distinguir cuatro categorías principales: a) la copa de los árboles; b) los espacios abiertos verdes; c) los techos verdes, y d) los sistemas verticales de vegetación (4). A su vez, los sistemas verticales de vegetación pueden agruparse en fachadas verdes con plantas trepadoras o muros verdes con plantas cultivadas sobre estructuras de soporte integradas a las paredes externas del edificio (5). La aplicación de fachadas verdes es una característica establecida del diseño

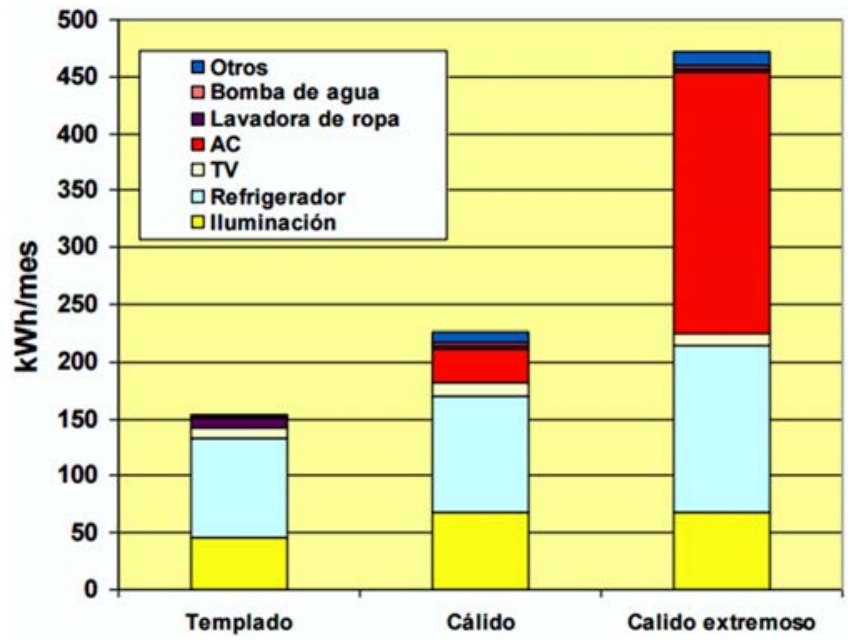

Figura 2. Estimados de usos finales de electricidad por zonas climáticas. AC (aire acondicionado). Fuente: (3).

urbano contemporáneo, y puede ofrecer múltiples beneficios ambientales tanto en edificios nuevos como en edificios existentes, además puede ser un enfoque sostenible en términos de ahorro de energía considerando materiales utilizados, nutrientes, agua necesaria y preservación eficiente de edificios (6).

Las barreras verdes son otro tipo de sistemas de vegetación que pueden ser empleados en bioclimatización. Estos sistemas, también llamados barreras vivas, son arreglos lineales para el establecimiento de especies vegetales destinadas a minimizar la velocidad del viento, conservar la humedad, delimitar terrenos, proporcionar sombra y mejorar el paisaje (7). En un principio su función primordial era la protección contra el viento, actualmente también se usan como amortiguadores acústicos. En este trabajo se plantea la posibilidad de aprovechar su capacidad de proporcionar sombra para usarlo como sistemas de amortiguamiento térmico para edificaciones.

Las barreras verdes presentan las desventajas de que, al usar especies vegetales arbóreas y arbustivas, requieren suficiente espacio para su instalación y tardan periodos relativamente largos de tiempo para crecer y proporcionar la protección requerida. Sin embargo, si se eligen especies de pastos de rápido crecimiento, como el bambú o el carrizo, es posible reducir

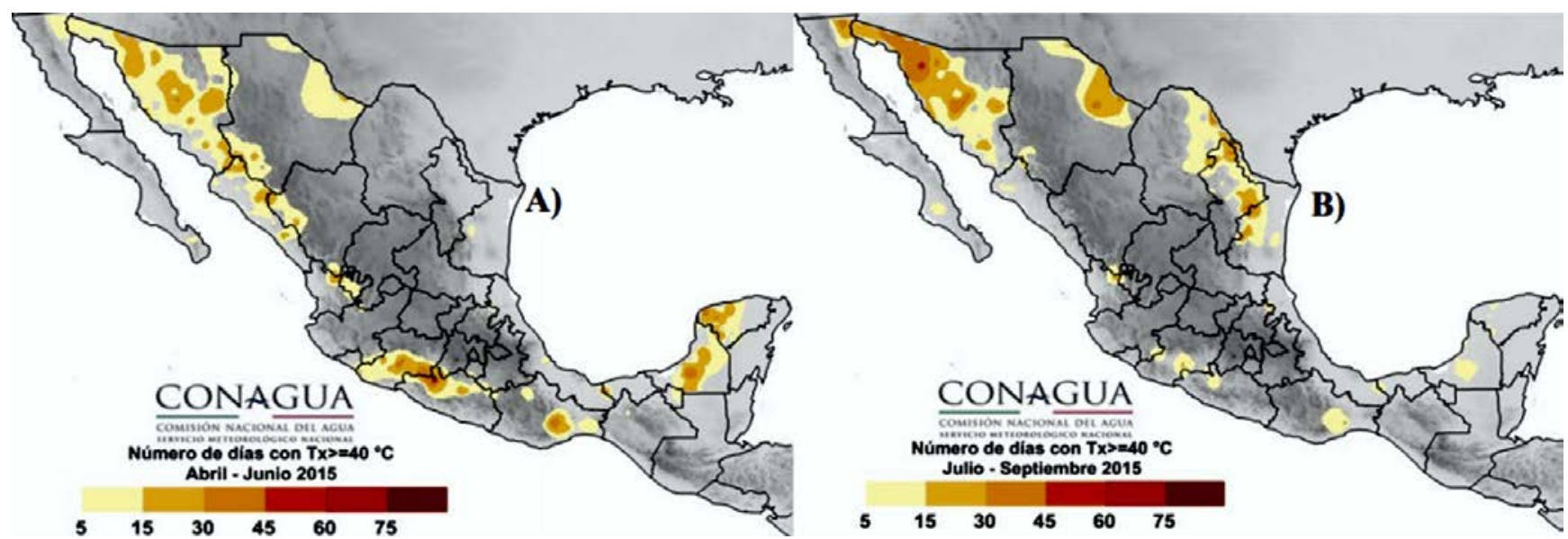

Figura 1. A) Frecuencia de días con temperatura máxima diaria mayor o igual a $40^{\circ}$ de abril a junio. B) Frecuencia de días con temperatura máxima diaria mayor o igual a $40^{\circ}$ de julio a septiembre. Fuente: (1). 
el tiempo de cobertura. Además, en tal caso su geometría más o menos regular contribuye a la reducción del espacio requerido para su instalación.

El presente trabajo propone utilizar, como barrera verde, una especie vegetal conocida como carrizo, caña gigante o falso bambú, cuyo nombre científico es Arundo donax, debido a las ventajas que ofrece en cuanto a tiempo de crecimiento y necesidades de agua y nutrientes para su mantenimiento. Esta especie tiene la capacidad de adaptarse a suelos de baja calidad, tiene tolerancia a suelos ácidos y alcalinos $(5 \leq \mathrm{pH} \leq 8,7)$, tiene una flexibilidad fisiológica que le permite desarrollarse en áreas con precipitaciones pluviales que van desde los 300 $\mathrm{mm}$ hasta los $4.000 \mathrm{~mm}$ anuales, y tiene además capacidad para sobrevivir en áreas de alta salinidad (8).

El Arundo donax tiene gran potencial de crecimiento y alta productividad de biomasa, lo que lo posicionan como una de las especies con mayor rango de desarrollo y producción primaria en todo el planeta. Se ha registrado que, en condiciones óptimas, el Arundo donax crece hasta $5 \mathrm{~cm}$ por día (8). Además, su gran cantidad de biomasa aérea proporciona aislamiento térmico en invierno (9).

El uso de Arundo donax en construcción se ha limitado a emplear sus tallos en sustitución de madera y, debido a su baja conductividad térmica, actúa como aislante térmico en construcciones rurales tradicionales (10).

El modelo de barrera verde de Arundo donax puede aportar mayores beneficios que las fachadas verdes y los muros vivos. Las fachadas verdes, en su mayoría, requieren de varios años para cubrir completamente la superficie de las paredes, y algunas especies pueden dañar los muros con la intrusión de sus raíces; otras especies requieren estructura de sostén. Los muros vivos requieren la instalación de materiales de sustrato, estructura de sostén e instalaciones para riego que incrementan los costos de instalación y mantenimiento, así como el impacto ambiental derivado del uso de recursos materiales, energéticos y agua. En ambos casos el espesor de la vegetación está limitado por el crecimiento propio de las especies utilizadas y por el peso que puede soportar el edificio o la estructura donde se instale.

Adicionalmente, el Arundo donax es una especie ampliamente utilizada en humedales artificiales (11) (12) (13) (14). Es tolerante a cargas orgánicas y nutrientes elevados, posee raíces y rizomas bien desarrollados, que ofrecen sustrato a las bacterias adheridas y oxigenación a las zonas adyacentes (9). Estudios previos demuestran que la tasa de producción de biomasa del Arundo donax se incrementa al regarse con agua residual (15) (16).

En este trabajo se plantea la posibilidad de, aprovechando las características antes descritas, usar la barrera verde de Arundo donax para amortiguamiento térmico en edificios, como estrategia alternativa que permita disminuir el consumo energético asociado a la necesidad de climatización de los edificios, al mismo tiempo que contribuye a la fijación de carbono en la biomasa y aprovechamiento del agua residual para su crecimiento.

Lo anterior derivado de que el uso de sistemas de vegetación vertical, como elementos de bioclimatización, es cada vez más común en la arquitectura moderna (5). Como parte de estos sistemas las fachadas verdes influyen positivamente en la sostenibilidad de los edificios y en el ahorro energético, pues disminuyen el efecto de calentamiento y mitigan la radiación solar (17). Sin embargo, existe una escasez de información sobre su contribución en entornos de edificios y el rendimiento energético en climas secos (18).

\section{MATERIALES Y MÉTODOS}

Hasta ahora el conocimiento sobre el efecto de sistemas de vegetación vertical sobre el comportamiento térmico de las fachadas aisladas es limitado (19). No se encontró reportada alguna metodología específica para determinar el amortiguamiento térmico por barreras verdes, ya que las identificadas se enfocan en muros vivos, fachadas verdes (17) y techos verdes (20). Un enfoque metodológico encontrado en los estudios de fachadas y muros verdes es la determinación de las temperaturas superficiales de los muros con y sin vegetación (5) (21) (22) y realizar una comparación entre ambos (23). Otros incluyen adicional a las mediciones de temperaturas la determinación de la transpiración, tal es el caso de un estudio realizado en tres fachadas de edificios en Berlín, Alemania, en el cual se determinaron las tasas de transpiración y las temperaturas superficiales de las paredes verdes y desnudas en periodos calurosos de verano (24).

En cuanto a las especies estudiadas destacan las plantas trepadoras Fuchsia, Jasminum y Lonicera (25), Parthenocissus tricuspidata, Hedera helix y Fallopia baldschuanica (24), Pueraria lobata (26), usadas tradicionalmente en fachadas verdes. No se encontraron datos acerca de Arundo donax o especies semejantes.

Respecto a las características del sitio donde se han realizado los estudios, se identificaron dos tendencias, la primera se refiere a edificios reales con fachadas verdes (22) (27) (28), y la segunda es mediante la implementación de cubículos o edificios prototipos en los que se instala el sistema de vegetación vertical específicamente para el estudio (5) (19) (29) (30) (31).

Derivado de lo anterior, en la presente investigación se realizó la medición de temperatura interior (ti), temperatura exterior (Te), masa aérea húmeda de la barrera verde, volumen aéreo de la barrera verde y superficie foliar de la barrera verde, para determinar el amortiguamiento térmico (Te - ti), y transpiración de una barrera verde de Arundo donax tipo. Para lo cual se utilizaron calorímetros prototipo construidos dentro de un invernadero ubicado en México en el Ejido de San José el Vidrio, Municipio de Nicolás Romero, Estado de México, coordenadas $19^{\circ} 38^{\prime} 58.93^{\prime} \mathrm{N}, 99^{\circ} 20^{\prime}$ 45.73" W y 2.517 metros sobre el nivel del mar, y la cámara de condiciones controladas del laboratorio de termofluidos de la Universidad Autónoma Metropolitana, campus Atzcapotzalco.

\subsection{Determinación de amortiguamiento térmico}

Se construyeron 2 calorímetros de forma cúbica $\left(1 \mathrm{~m}^{3}\right)$, las dimensiones seleccionadas facilitan los cálculos y comparaciones posteriores con otros sistemas de aire acondicionado. Uno de los calorímetros se usó de testigo y el otro de prueba. Ambos fueron construidos con 5 paredes hechas de poliestireno expandido (espesor: $\mathrm{lps}=0,2 \mathrm{~m}$ y densidad 
$\rho p s=16 \mathrm{~kg} / \mathrm{m}^{3}$ ), que sirven como aislante térmico. Estas 5 caras fueron cubiertas exteriormente con lámina de aluminio (calibre: lal $=1,7 \times 10^{-5} \mathrm{~m}$ ). En la sexta pared, orientada hacia el este, se utilizó una lámina de papel (calibre: lp = $2,5 \times 10^{-4} \mathrm{~m}$, densidad $\rho \mathrm{p}=626,28 \mathrm{~kg} / \mathrm{m}^{3}$ ) que permite la conducción de calor. Se cubrió el cubo completo con una película de polietileno (calibre: lpe $=2,3 \times 10^{-4} \mathrm{~m}$, densidad $\rho p e=141,10 \mathrm{~kg} / \mathrm{m}^{3}$ ) para evitar el intercambio de aire con el exterior del sistema (Figura 3).

Los calorímetros se colocaron dentro de un invernadero ( $\mathrm{Fi}$ gura 4) para determinar el comportamiento de la barrera verde en condiciones controladas de humedad y temperatura características de climas cálidos.
En la pared orientada hacia el este del calorímetro de prueba se colocó una barrera verde de Arundo donax de 0,4 m de espesor y densidad de población de 120 tallos $/ \mathrm{m}^{2}$ (Figura 5). Se construyó la barrera verde empleando ejemplares de Arundo donax obtenidos de un humedal doméstico con tallos de 1,2 m de largo. Se utilizó Tezontle como sustrato. Se midieron las temperaturas en tres horarios por día, 8:30, 13:30 y 17:00 horas. Las mediciones se realizaron del 27 de julio al 30 de septiembre del 2015. Se midieron las temperaturas al interior (ti) y al exterior (Te) de los calorímetros (Figuras 3 y 5), para ello se utilizó un termómetro con las especificaciones siguientes: intervalo de temperatura interior o a $50^{\circ} \mathrm{C}$, intervalo de temperatura exterior -20 a $50^{\circ} \mathrm{C}$, intervalo de compensación de temperatura $-10^{\circ} \mathrm{C}$
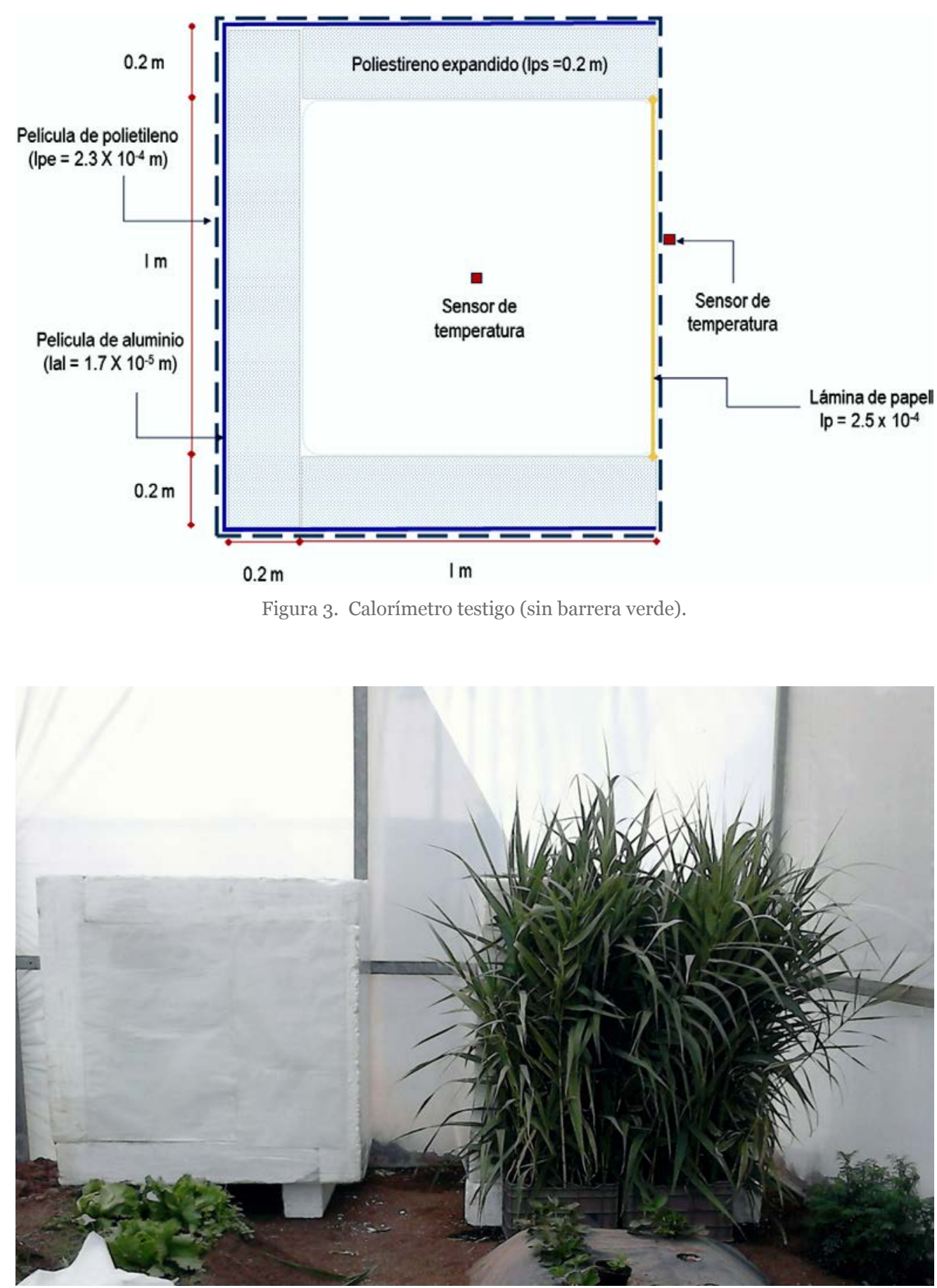

Figura 4. Calorímetros al interior del invernadero. 


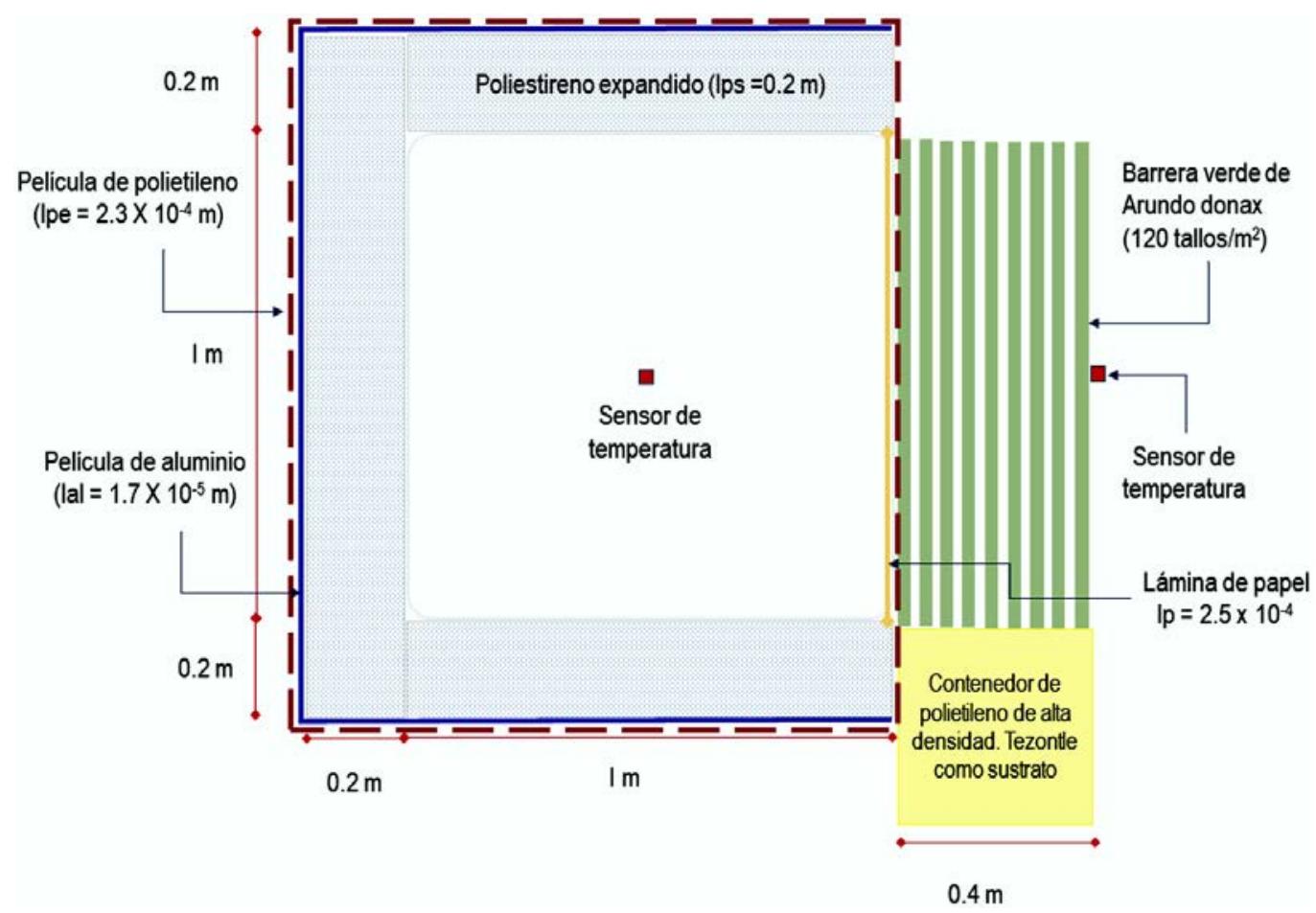

Figura 5. Calorímetro de prueba (con barrera verde).

a $60^{\circ} \mathrm{C}$, medición mínima de $0,1^{\circ} \mathrm{C}$, frecuencia del Sensor RF $433 \mathrm{MHz}$.

\subsection{Determinación de la transpiración}

Se realizaron pruebas en una cámara de condiciones controladas, donde se introdujeron ejemplares de Arundo donax y se sometieron a una temperatura de $45^{\circ} \mathrm{C}$ y $13 \%$ de humedad durante un periodo de 2 horas. El sustrato de los ejemplares se saturó con humedad para garantizar el abasto de agua hacia la planta, adicionalmente se envolvió el recipiente con dicho sustrato con una película de polietileno para evitar la evaporación desde el sustrato, de tal manera que sólo se cuantificara la transpiración de las plantas. Asimismo se determinó la superficie foliar de una población de Arundo donax con densidad de población de 120 tallos $/ \mathrm{m}^{2}$ y alimentada sin restricciones con agua residual gris doméstica. Con la finalidad de contar con un punto de comparación se realizó una prueba de evaporación de agua en iguales condiciones ambientales.

\section{RESULTADOS}

\subsection{Amortiguamiento térmico}

Las temperaturas medidas al interior del calorímetro con barrera verde fueron menores que las encontradas en el calorímetro sin barrera verde. En la Tabla 1a se muestran las temperaturas máximas y mínimas medidas al exterior y al interior de ambos dispositivos.

El promedio del amortiguamiento térmico ( $\mathrm{Te}$ - ti) en el dispositivo con barrera verde fue de $7,5^{\circ} \mathrm{C}$, y en el dispositivo sin barrera verde fue de $5,0^{\circ} \mathrm{C}$. En la Tabla $1 \mathrm{~b}$ se muestran los promedios de amortiguamiento térmico para cada horario de mediciones (Figura 6). Para el dispositivo con barrera verde el amortiguamiento térmico es mayor en el horario de las 13:30 horas, es decir, en el horario de mayor temperatura ambiental. En el horario de las 17:00 el amortiguamiento térmico es menor por el calor almacenado durante el día. En el dispositivo sin barrera verde se observa menor capacidad

Tabla 1a. Comparación de temperatura interior y exterior mínima, promedio y máxima.

\begin{tabular}{|c|c|c|c|c|}
\hline Ubicación & $\underset{(\mathrm{O} C)}{\operatorname{Tmin}}$ & $\begin{array}{c}\text { Tprom. } \\
\text { (o C) }\end{array}$ & $\underset{\left({ }^{\circ} \mathrm{C}\right)}{\operatorname{Tmax}}$ & $\begin{array}{c}\mathrm{T}>\mathbf{3 0}_{(\%)}^{\mathbf{3 0}^{\circ} \mathrm{C}} \\
\text { (\%) }\end{array}$ \\
\hline Exterior de los dispositivos & 12,2 & 29,4 & 49,9 & 51,3 \\
\hline Interior del dispositivo con barrera verde & 10,2 & 21,5 & 34,9 & 10,0 \\
\hline Interior del dispositivo sin barrera verde & 10,3 & 24,7 & 44,1 & 30,0 \\
\hline
\end{tabular}

Tabla 1b. Comparación de amortiguamiento térmico con y sin barrera verde.

\begin{tabular}{|l|c|c|c|c|}
\hline \multirow{2}{*}{$\begin{array}{c}\text { Promedio por horario } \\
\text { y promedio general }\end{array}$} & \multicolumn{3}{|c|}{ Amortiguamiento térmico (Te - ti) (o C) } \\
\cline { 2 - 5 } & $\mathbf{8 : 3 0} \mathbf{~ h}$ & $\mathbf{1 3 : 3 0} \mathbf{~ h}$ & $\mathbf{1 7 : 0 0} \mathbf{~ h}$ & Promedio general \\
\hline Con barrera verde & 6,0 & 11,4 & 5,2 & 7,5 \\
\hline Sin barrera verde & 5,6 & 7,2 & 2,2 & 5 \\
\hline
\end{tabular}




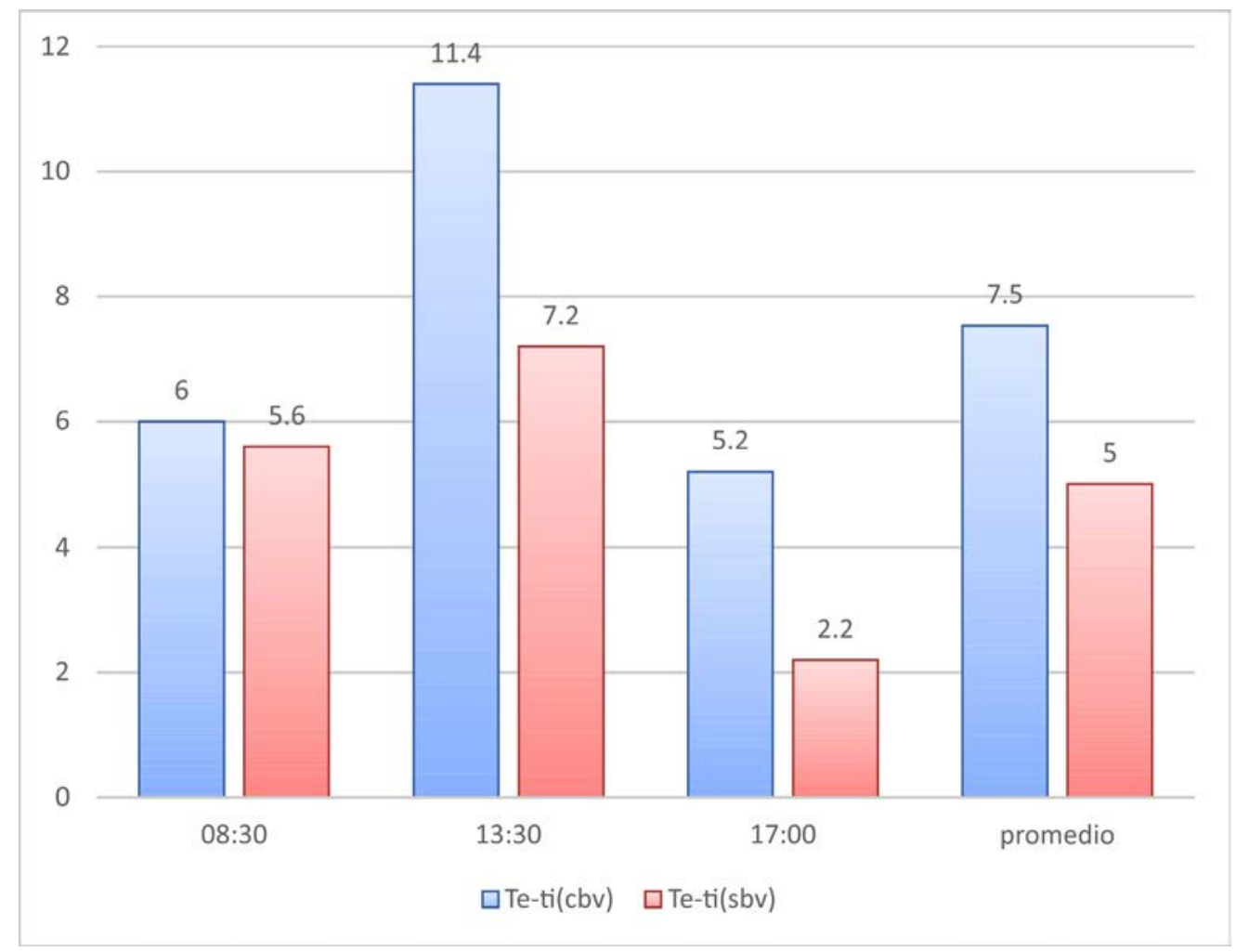

Figura 6. Amortiguamiento térmico promedio para cada horario de medición. Comparación del dispositivo con barrera verde [Te - ti(cbv)] y el dispositivo sin barrera verde [Te-ti(sbv)].

de amortiguamiento térmico en el horario de las 17:00, lo que significa que el calor almacenado durante el día es mayor que en el caso del dispositivo con barrera verde.

Se realizó el análisis de los datos de temperatura mediante el software MINITAB-17. Con la prueba de Anderson-Darling se verificó la normalidad de los datos. Al tratarse de un experimento con dos variables, una continua (temperatura) y otra discreta (presencia o ausencia de barrera verde), se empleó un Análisis de Varianza (ANOVA) de una vía mediante el cual se rechazó la hipótesis nula: «la media de las mediciones de temperatura son iguales». Se aceptó la hipótesis alterna: «existe por lo menos uno diferente», lo que significa que sí existe una diferencia estadísticamente significativa entre los valores de temperatura obtenidos para los casos que hay presencia y ausencia de barrera verde.

En la Figura 7 se muestra la termografía realizada en ambos casos, con y sin barrera verde. Asimismo se observa la gráfica correspondiente a los porcentajes de superficie con diferente temperatura.
En la Tabla 2 se muestra un comparativo entre los resultados de estudios llevados a cabo con diversas especies y los obtenidos con Arundo donax en esta investigación. Cabe señalar que, aunque en todos ellos se realizaron mediciones de temperatura con y sin vegetación, el punto exacto de la medición varía.

\subsection{Transpiración}

La transpiración promedio del Arundo donax fue de 4,4 l/ $\mathrm{m}^{3} \cdot \mathrm{h}$ en condiciones ambientales de $45^{\circ} \mathrm{C}$ y $13 \%$ de humedad relativa. Este valor de transpiración corresponde a un volumen de $1 \mathrm{~m}^{3}$ de barrera verde, con superficie foliar de $28 \mathrm{~m}^{2}$, caracterizada para una población de Arundo donax de 120 tallos $/ \mathrm{m}^{2}$ sin restricciones de riego. Es importante mencionar que dichas condiciones ambientales extremas sólo se alcanzan durante unas pocas horas al medio día, unos cuantos días al año en la época más calurosa (abril a septiembre) (1) en clima cálido seco. En comparación, la evaporación en iguales condiciones ambientales resultó de $1,7 \mathrm{~mm} / \mathrm{h}$.
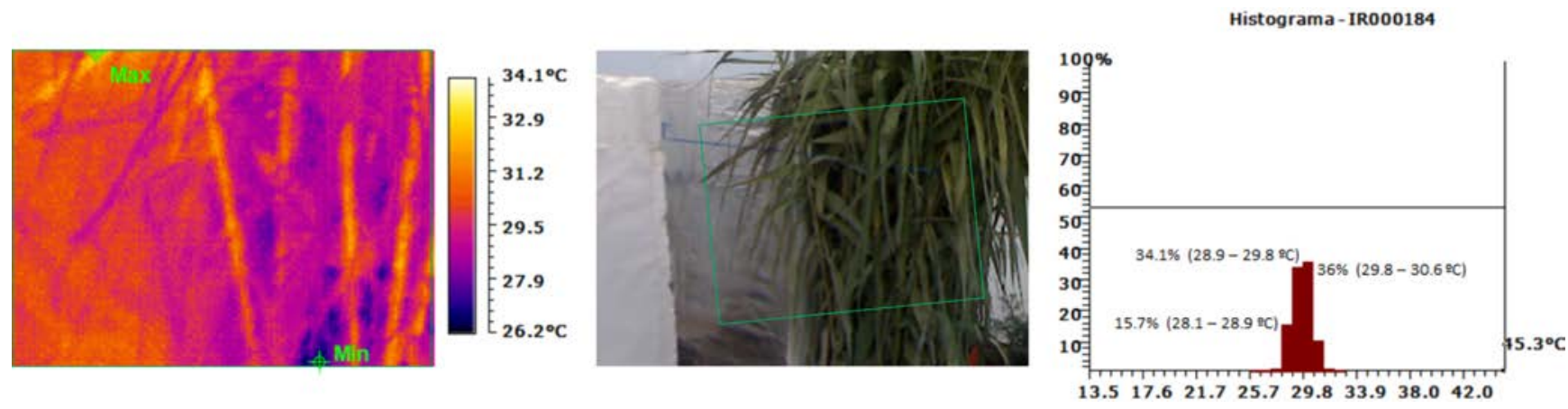

Figura 7. Termografía de la pared con y sin barrera verde de Arundo donax. 
Tabla 2. Comparación de efectos sobre la temperatura de los muros de los sistemas de vegetación vertical con diferentes especies.

\begin{tabular}{|c|c|c|}
\hline $\begin{array}{l}\text { Sistema de } \\
\text { vegetación vertical }\end{array}$ & Especies & Variable medida y resultados \\
\hline Fachadas verdes (24) & $\begin{array}{l}\text { Parthenocissus tricuspidata, Hedera } \\
\text { hélix, Fallopia baldschuanica }\end{array}$ & $\begin{array}{l}\text { Temperatura superficial exterior: Disminución de } 15,5^{\circ} \mathrm{C} \\
\text { Temperatura superficial interior: Disminución de } 1,7^{\circ} \mathrm{C} \\
\end{array}$ \\
\hline Fachadas verdes (27) & - & Temperatura interior: Disminución de $5^{\circ} \mathrm{C}$ en promedio \\
\hline Fachadas verdes (25) & $\begin{array}{l}\text { Arbustos de pared y plantas trepadoras: } \\
\text { Prunus laurocerasus } \\
\text { Stachys y Hedera }\end{array}$ & $\begin{array}{l}\text { Temperatura del aire adyacente a las paredes en ambientes controlados } \\
\text { - Disminución de } 6,3^{\circ} \mathrm{C} \\
\text { - Disminución }>7,0^{\circ} \mathrm{C}\end{array}$ \\
\hline Fachada verde (26) & Pueraria lobata & Reducción máxima de temperatura ambiente: $3,44^{\circ} \mathrm{C}$ \\
\hline Fachada verde (5) & $\begin{array}{l}\text { Hedera hélice } \\
\text { (10 cm de espesor) }\end{array}$ & Reducción en la temperatura interna de la pared: $2,5^{\circ} \mathrm{C}$ \\
\hline Muro verde interior (32) & - & $\begin{array}{l}\text { Reducción media de la temperatura ambiente: } 4^{\circ} \mathrm{C} \\
\text { Reducción máxima de la temperatura ambiente: } 6^{\circ} \mathrm{C}\end{array}$ \\
\hline $\begin{array}{l}\text { Muro verde } \\
\text { Fachada verde (5) }\end{array}$ & - & $\begin{array}{l}\text { Reducción de la temperatura interior: } 4,0^{\circ} \mathrm{C}(\mathrm{Fv}) \\
\text { Reducción de la temperatura interior: } 3,0^{\circ} \mathrm{C}(\mathrm{Mv})\end{array}$ \\
\hline Barreras verdes* & Arundo donax & $\begin{array}{l}\text { Diferencia entre la temperatura interior y exterior } \\
\text { Con barrera verde: } 7,5^{\circ} \mathrm{C} \\
\text { Sin barrera verde: } 5,0^{\circ} \mathrm{C}\end{array}$ \\
\hline
\end{tabular}

* Datos experimentales.

\section{DISCUSIÓN DE RESULTADOS}

La temperatura promedio en el dispositivo con barrera verde $\left(21,2^{\circ} \mathrm{C}\right)$ se encuentra dentro del intervalo considerado de confort, $20-24^{\circ} \mathrm{C}$ de acuerdo con la localización geográfica y el clima de la zona (33), no así el dispositivo sin barrera verde y el exterior, ambos con valores por encima del confort (Tabla 1a). En el dispositivo con barrera verde la variación de temperatura (Tmax - Tmin) es menor que en el dispositivo sin barrera verde y en el exterior. En promedio, el amortiguamiento térmico en el dispositivo con barrera verde es mayor que en el dispositivo sin barrera verde. La diferencia mayor en el horario de las 13:30 horas demuestra la capacidad de la barrera verde de modificar el nivel de amortiguamiento de acuerdo con la situación ambiental en la que se encuentre.

En la comparación con los resultados de otros estudios (ver Tabla 2) se observa que con la barrera verde de Arundo donax se obtuvieron valores de disminución de temperatura ligeramente superiores a los encontrados con las especies Hedera $\left(7,0^{\circ} \mathrm{C}\right)$ y Prunus laurocerasus, la cual, también proporcionó un enfriamiento significativo de la pared en ambientes controlados $\left(6,3^{\circ} \mathrm{C}\right)(25)$. Es importante resaltar esta última, ya que, al tratarse de una especie arbustiva, este sistema de vegetación vertical es el que más se asemeja con el sistema de barrera verde empleado en el presente estudio.

En cuanto a los mecanismos para proporcionar el enfriamiento de las paredes éstos varían entre las diferentes especies (25). Dentro de los más importantes se puede distinguir la refrigeración por sombreamiento y la refrigeración por transpiración. Esta última depende de factores ambientales como humedad de la planta, temperatura ambiente, humedad del ambiente, velocidad del viento y radiación (34). También intervienen factores inherentes a la planta tales como la fisiología, el área foliar y la morfología foliar, los cuales deben ser considerados al seleccionar especies para maximizar el enfriamiento en aplicaciones de muros verdes (25). La superficie foliar está directamente ligada al suministro de agua, es decir, cuando existen restricciones de riego el mecanismo de la planta es evitar la transpiración deshaciéndose de las hojas (ver Tabla 3). Garantizar el abasto de agua dará como consecuencia una mayor cantidad de hojas, que en condiciones de temperaturas elevadas y valores de humedad relativa bajos promoverán la transpiración.

En el caso del Arundo donax al ser la transpiración determinada en condiciones ambientales extremas se observó un gran potencial para emplear esta especie en sitios con clima cálido seco, donde las necesidades de riego dificultarían el uso de otras especies. La ventaja de esta especie radica en la posibilidad de ser regada con agua residual gris proveniente de descargas domésticas.

En cuanto al efecto de refrigeración por sombreamiento, éste puede ser aprovechado para diversos tipos de clima mediante la combinación de las variables: orientación del edificio, altura del edificio, ubicación de la barrera verde y altura de la misma. En la Figura 8 se muestra una configuración que puede ser utilizada en un clima cálido seco, en el cual los beneficios del sistema consisten en máximo de horas de sombra en la mañana y en la tarde, y pocas horas de sol al medio día sólo en el techo.

Tabla 3. Comparación de humedad y proporción de tallos y hojas en individuos de Arundo donax sin restricciones de agua y con restricciones de agua.

\begin{tabular}{|c|c|c|c|c|}
\hline \multirow{2}{*}{$\begin{array}{l}\text { Parte de la } \\
\text { planta }\end{array}$} & \multicolumn{2}{|c|}{ Humedal doméstico* } & \multicolumn{2}{|c|}{ Población silvestre ${ }^{* *}$} \\
\hline & $\begin{array}{c}\text { Proporción }^{* * *} \\
\text { (\%) }\end{array}$ & Humedad (\%) & $\begin{array}{c}\text { Proporción }^{* * *} \\
\text { (\%) }\end{array}$ & Humedad (\%) \\
\hline Hojas & 45,95 & 64,05 & 23,28 & 55,71 \\
\hline Tallo & 54,05 & 53,81 & 76,72 & 57,30 \\
\hline Total & & 58,59 & & 58,63 \\
\hline
\end{tabular}

* Población de Arundo donax sin restricciones de agua.

** Población de Arundo donax con restricciones de agua.

*** La proporción se determinó a partir de la masa húmeda de tallos, hojas y planta completa. 


\section{Clima Cálido Seco}

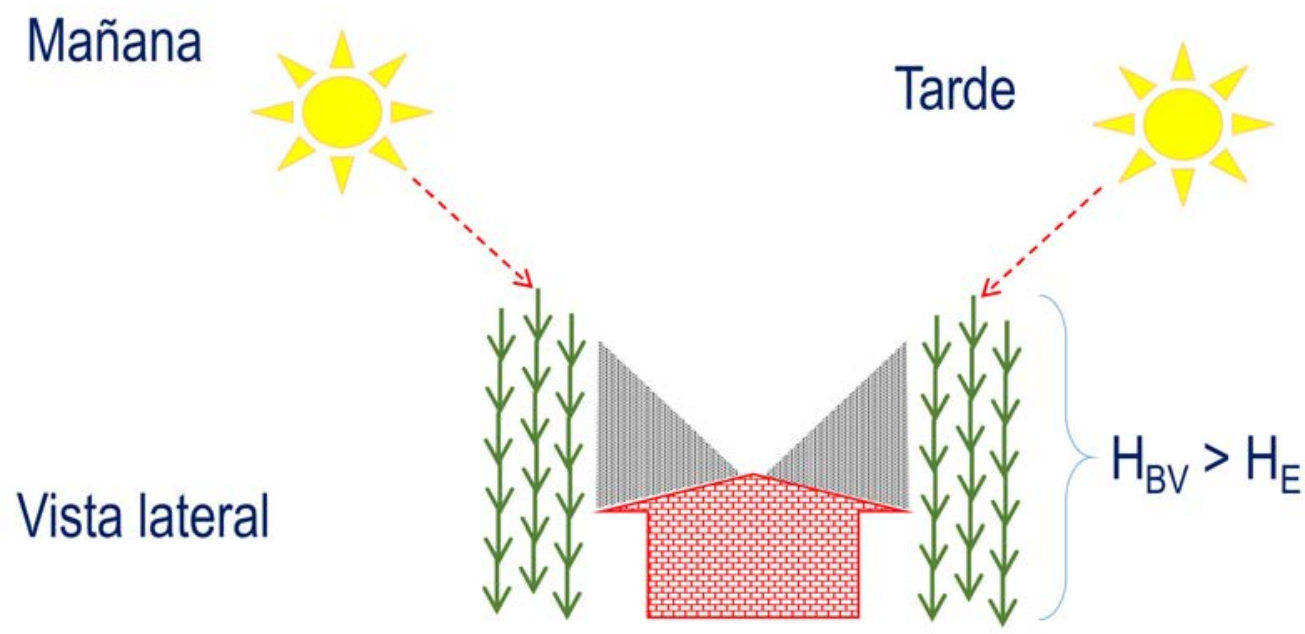

\section{Vista superior}

\author{
Hbv: altura de la barrera verde
} He: altura del edificio.

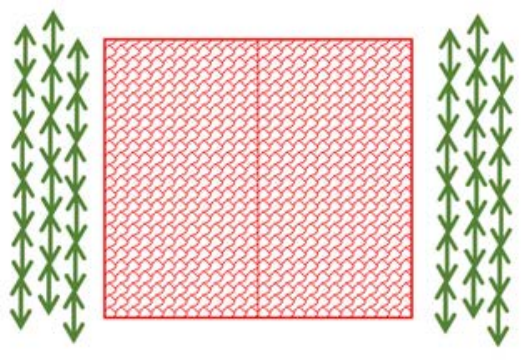

Figura 8. Configuración de la barrera verde en función de la orientación y altura del edificio, recomendada para clima cálido seco.

\section{CONCLUSIONES}

El sistema de barrera verde de Arundo donax posee características térmicas susceptibles de ser aprovechadas para contribuir a la eficiencia energética de los edificios tales como la estrategia pasiva de refrigeración por sombreamiento, además de la estrategia dinámica de refrigeración por transpiración, propia de los organismos vegetales.

La barrera verde puede tener una utilidad importante en diversas regiones de México, con climas extremosos, especialmente en el norte del país donde los climas son secos. La baja humedad en el ambiente propicia la transpiración de las plantas y con esto la refrigeración por transpiración. Debido a que la barrera verde emplea agua residual para su mantenimiento, aporta una ventaja adicional al no consumir agua potable y evitar la contaminación derivada de la descarga del agua residual sin tratamiento. Asimismo conlleva el ahorro de energía por concepto de climatización y se captura carbono en la biomasa de la barrera verde. Por otra parte, una limitación que presenta este sistema es la altura del edificio que puede proteger, ya que el Arundo donax, en condiciones climáticas favorables, puede alcanzar 9-10 $\mathrm{m}$ de altura, sin embargo, se le encuentra mayormente con altura de 5-6 m. Además se debe tener en cuenta la necesidad de poda regular debido a su alta productividad de biomasa.

Por todo ello, además de ser un elemento bioclimático, la barrera verde de Arundo donax puede representar una alternativa integral para disminuir el impacto ambiental derivado de la operación de edificios residenciales por la generación de agua residual gris y la emisión de gases de efecto invernadero.

Dada la importancia de la transpiración en el comportamiento térmico de la barrera verde se identificó la necesidad de realizar investigaciones posteriores enfocadas a establecer la contribución individual de cada mecanismo de refrigeración (sombreamiento y transpiración). Lo anterior permitirá determinar con mayor precisión las regiones y los periodos estacionales donde la implementación de este modelo de barrera verde puede ser de mayor utilidad. 


\section{REFERENCIAS}

(1) CONAGUA (2014). Reporte del clima en México. Reporte anual 2014. Coordinación General del Servicio Meteorológico Nacional, Gerencia de Meteorología y Climatología, Subgerencia de Pronóstico a Mediano y Largo Plazo. http://smn1. conagua.gob.mx/climatologia/analisis/reporte/Anual2014.pdf

(2) Garrido, L. (2014). Arquitectura Bioclimática Extrema, p. 19. Barcelona: MONSA.

(3) Odon de Buen (2011, junio). Eficiencia energética y energías renovables en la vivienda en México: Un Repaso, http:// mexiko.ahk.de/fileadmin/ahk_mexiko/news_bilder/9_ENTE_Ing._Odon_de_Buen.pdf

(4) Koc, C. B., Osmond, P., Peters, A. (2017). Towards a comprehensive green infrastructure typology: a systematic review of approaches, methods and typologies. Urban Ecosystems, 20(1): 15-35, doi: http://dx.doi.org/10.1007/s11252-0160578-5.

(5) Cuce, E. (2017). Thermal regulation impact of green walls: An experimental and numerical investigation. Applied Energy, 194: 247-254, doi: http://dx.doi.org/10.1016/j.apenergy.2016.09.079

(6) Perini, K., Ottelé, M., Haas, E. M., Raiteri, R. (2013). Vertical greening systems, a process tree for green façades and living walls. Urban Ecosystems, 16(2): 265-277, doi: http://dx.doi.org/10.1007/s11252-012-0262-3.

(7) Fernández, D. S., Martínez, M. R., Ramírez, M. L. (2009). Prácticas vegetativas y agronómicas complementarias al proyecto integral. Texcoco, Estado de México: Colegio de Posgraduados Sagarpa, Subsecretaría de Desarrollo Rural, Dirección General de Apoyos para Desarrollo Rural.

(8) Flores, J., Prado, A, Domínguez, A. L., Mendoza, R., González, A. (2008) El carrizo gigante, especie invasora de ecosistemas riparios. Biodiversitas (81): 1870-1760.

(9) Vymazal, J. (2011). Plants used in constructed wetlands with horizontal subsurface flow: a review. Hydrobiologia, 674(1): 133-156, doi: http://dx.doi.org/10.1007/s10750-011-0738-9.

(10) Barreca, F. (2012). Use of giant reed Arundo Donax L. in rural constructions. Agricultural Engineering International: CIGR Journal, 14(3): 46-52.

(11) Tzanakakis, V. E., Paranychianakis, N. V., Angelakis, A. N. (2007). Performance of slow rate systems for treatment of domestic wastewater. Water Science \& Technology, 55(1): 139-147.

(12) Sabeen, M., Mahmood, Q., Irshad, M., Fareed, I., Khan, A., Ullah, F., ..., Tabassum, S. (2013). Cadmium phytoremediation by Arundo donax L. from contaminated soil and water. BioMed research international, 2013, doi: http://dx.doi. org/10.1155/2013/324830.

(13) Idris, S. M., Jones, P. L., Salzman, S. A., Croatto, G., Allinson, G. (2012). Evaluation of the giant reed (Arundo donax) in horizontal subsurface flow wetlands for the treatment of dairy processing factory wastewater. Environmental science and pollution research, 19(8): 3525-3537.

(14) Chang, J. J., Wu, S. Q., Dai, Y. R., Liang, W., Wu, Z. B. (2012). Treatment performance of integrated vertical-flow constructed wetland plots for domestic wastewater. Ecological Engineering, 44: 152-159.

(15) Mavrogianopoulos, G., Vogli, V., Kyritsis, S. (2002). Use of wastewater as a nutrient solution in a closed gravel hydroponic culture of giant reed (Arundo donax). Bioresource Technology, 82(2): 103-107.

(16) Barbagallo, S., Barbera, A. C., Cirelli, G. L., Milani, M., Toscano, A. (2014). Reuse of constructed wetland effluents for irrigation of energy crops. Water Science \& Technology, 70(9): 1465-1472.

(17) Campillo, L. M. M., Torres, D. (2013). Las fachadas verdes como herramienta pasiva de ahorro energético en el bloque administrativo de la Universidad Pontificia Bolivariana, seccional Montería. DEARQ: Revista de Arquitectura de la Universidad de los Andes (13): 140-149.

(18) Razzaghmanesh, M., Razzaghmanesh, M. (2017). Thermal performance investigation of a living wall in a dry climate of Australia. Building and Environment, 112: 45-62, doi: http://dx.doi.org/10.1016/j.buildenv.2016.11.023.

(19) Olivieri, F., Olivieri, L., Neila, J. (2014). Experimental study of the thermal-energy performance of an insulated vegetal façade under summer conditions in a continental mediterranean climate. Building and Environment, 77: 61-76, doi: http://dx.doi.org/10.1016/j.buildenv.2014.03.019.

(20) Neila. F. J., Bedoya, C., Acha, C., Olivieri, F., Barbero, M. (2008). Las cubiertas ecológicas de tercera generación: un nuevo material constructivo-The ecological roofts of third generation: an new constructive material. Informes de la Construcción, 60(511): 15-24.

(21) Cheng, C. Y., Cheung, K. K., Chu, L. M. (2010). Thermal performance of a vegetated cladding system on facade walls. Building and environment, 45(8): 1779-1787, doi: http://dx.doi.org/10.1016/j.buildenv.2010.02.005.

(22) Wong, N. H., Tan, A. Y. K., Chen, Y., Sekar, K., Tan, P. Y., Chan, D., ..., Wong, N. C. (2010). Thermal evaluation of vertical greenery systems for building walls. Building and environment, 45(3): 663-672, doi: http://dx.doi.org/10.1016/j. buildenv.2009.08.005.

(23) Tudiwer, D., Korjenic, A. (2017). The effect of living wall systems on the thermal resistance of the façade. Energy and Buildings, 135: 10-19, doi: http://dx.doi.org/10.1016/j.enbuild.2016.11.023.

(24) Hoelscher, M. T., Nehls, T., Jänicke, B., Wessolek, G. (2016). Quantifying cooling effects of facade greening: shading, transpiration and insulation. Energy and Buildings, 114: 283-290, doi: http://dx.doi.org/10.1016/j.enbuild.2015.06.047.

(25) Cameron, R. W., Taylor, J. E., Emmett, M. R. (2014). What's 'cool'in the world of green façades? How plant choice influences the cooling properties of green walls. Building and Environment, 73: 198-207, doi: http://dx.doi.org/10.1016/j. buildenv.2013.12.005.

(26) Koyama, T., Yoshinaga, M., Maeda, K. I., Yamauchi, A. (2014). Room temperature reductions in relation to growth traits of kudzu vine (Pueraria lobata): Experimental quantification. Ecological Engineering, 70: 217-226, doi: http://dx.doi. org/10.1016/j.ecoleng.2014.05.026. 
(27) Haggag, M., Hassan, A., Elmasry, S. (2014). Experimental study on reduced heat gain through green facades in a high heat load climate. Energy and Buildings, 82: 668-674, doi: http://dx.doi.org/10.1016/j.enbuild.2014.07.087.

(28) Yin, H., Kong, F., Middel, A., Dronova, I., Xu, H., James, P. (2017). Cooling effect of direct green façades during hot summer days: An observational study in Nanjing, China using TIR and 3DPC data. Building and Environment, 116: 195-206, doi: http://dx.doi.org/10.1016/j.buildenv.2017.02.020.

(29) Chen, Q., Li, B., Liu, X. (2013). An experimental evaluation of the living wall system in hot and humid climate. Energy and Buildings, 61: 298-307, doi: http://dx.doi.org/10.1016/j.enbuild.2013.02.030.

(30) Coma, J., Pérez, G., de Gracia, A., Burés, S., Urrestarazu, M., Cabeza, L. F. (2017). Vertical greenery systems for energy savings in buildings: A comparative study between green walls and green facades. Building and Environment, 111: 228237, doi: http://dx.doi.org/10.1016/j.buildenv.2016.11.014.

(31) Koyama, T., Yoshinaga, M., Maeda, K. I., Yamauchi, A. (2015). Transpiration cooling effect of climber greenwall with an air gap on indoor thermal environment. Ecological Engineering, 83: 343-353, doi: http://dx.doi.org/10.1016/j.ecoleng.2015.06.015.

(32) Cañero, R. F., Urrestarazu, L. P., Salas A. F. (2011). Assessment of the cooling potential of an indoor living wall using different substrates in a warm climate. Indoor and Built Environment, 21(5): 642-650. Actualizado Octubre 2012, doi: http://dx.doi.org/10.1177/1420326X11420457.

(33) Bernal, F., Ibargüengoitia, D. (2011). Confort ecológico. En Aguilar Dubose, C., Delgado Castillo, C. L. (Eds.), Diseño y construcción sostenibles: Realidad ineludible (pp. 105-108). México: Universidad Iberoamericana, Departamento de Arquitectura.

(34) Ángeles, V. (2002). Diseño agronómico de sistemas de riego presurizado, p. 64. México: Edit. Universidad Autónoma de Chapingo. 